\title{
Erratum to: Differential requirement of F-actin and microtubule cytoskeleton in cue-induced local protein synthesis in axonal growth cones
}

Michael Piper ${ }^{1,2}$, Aih Cheun Lee ${ }^{1,3}$, Francisca P.G van Horck', Heather McNeilly', Trina Bo Lu', William A Harris ${ }^{1}$ and Christine E Holt ${ }^{*}$

\section{Erratum}

We were recently made aware that one of the figures (Figure four (Fig. 1 here)) in our manuscript detailing the contribution of the cytoskeleton to cue-induced protein synthesis [1] contains a minor formatting error, introduced while the panels of the figures were being compiled. In panel I of the original Figure four (Fig. 1 here), we used white lines to divide the images of the growth cones that had been exposed to different cytoskeletal disrupting agents. However, the white lines were not placed appropriately, and as such, some of the filopodia from the control growth cones (the two left hand growth cones in panel I of Figure four (Fig. 1 here)) can be seen to the right of the dividing line. We have amended this figure to avoid any potential misinterpretation of the data.

\footnotetext{
*Correspondence: ceh33@cam.ac.uk

${ }^{1}$ Department of Physiology, Development and Neuroscience, University of Cambridge, Downing street, Cambridge CB2 3DY, UK

Full list of author information is available at the end of the article
} 


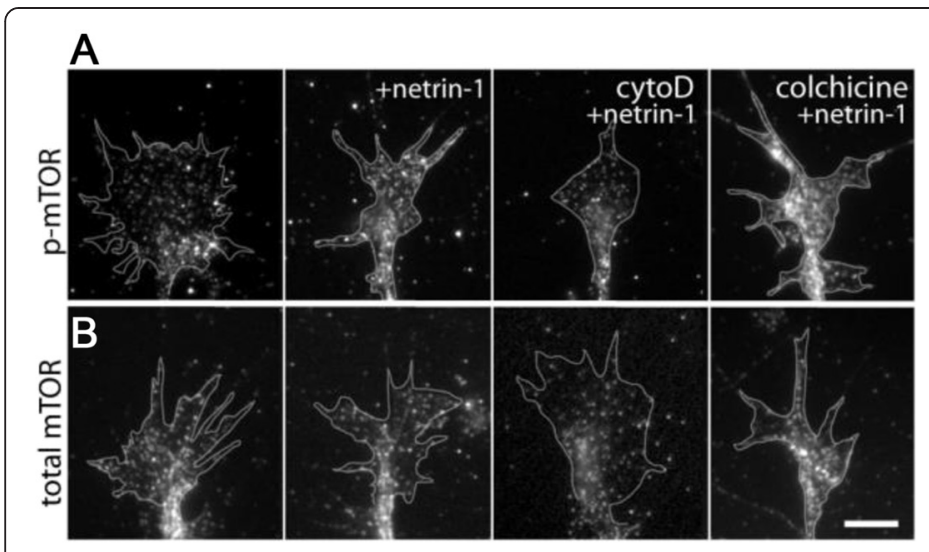

$$
\text { E }
$$

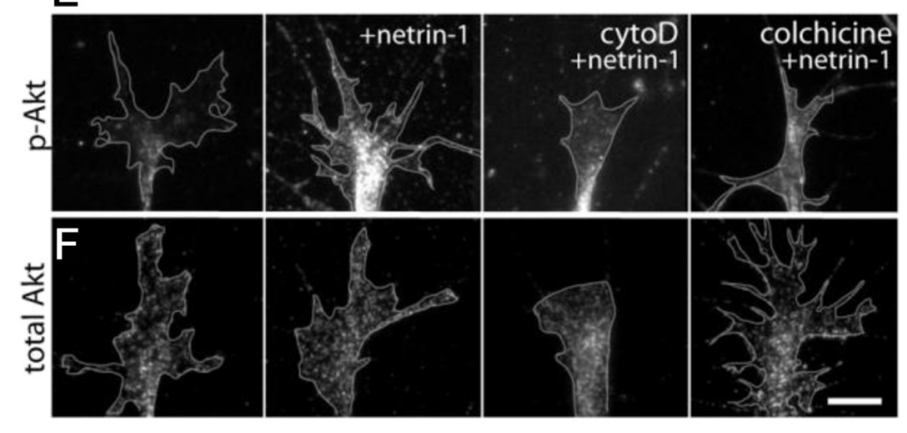

I

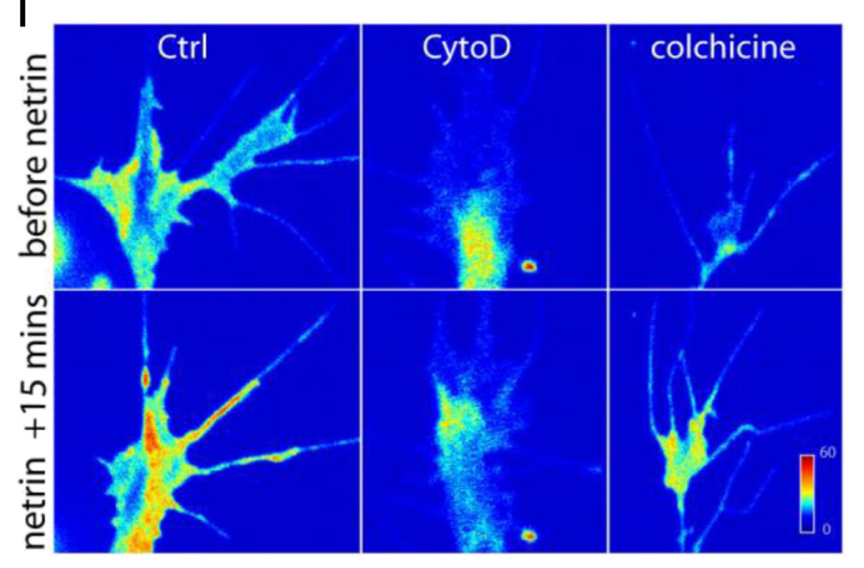

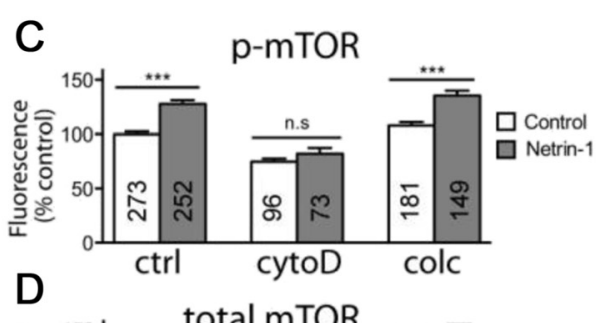
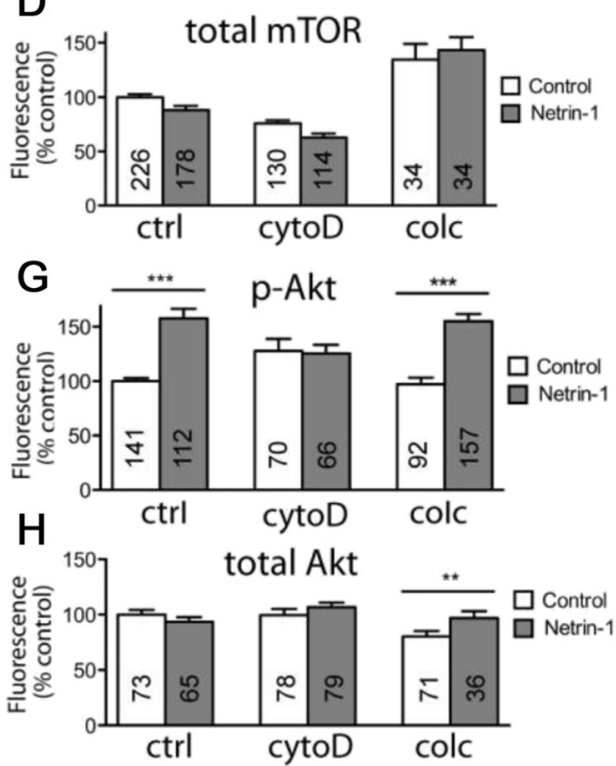

J

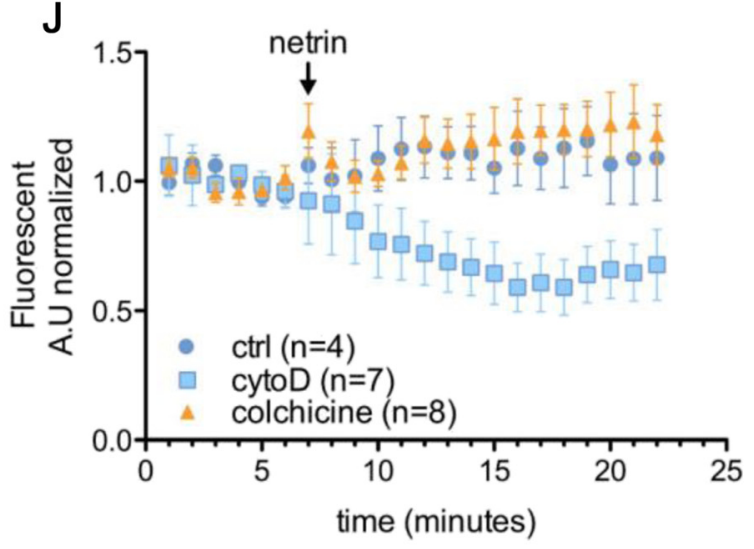

Fig. 1 Actin, but not microtubule, disruption blocks netrin-1 induced PI3K/Akt/mTOR signaling in growth cones. Cultured stage 24/25 retinal explants were treated with a DMSO vehicle control, cytochalasin D (CytD), or colchicine (Colc) for 5 min, followed by 5 -min stimulation with either control medium or netrin-1. Quantitative immunofluorescence showed that levels of activated mTOR ( $p$-mTOR; (a)) and Akt ( $p$-Akt; (e)) were elevated by netrin-1 stimulation, a process that was prevented by actin, but not microtubule disruption (quantified in (c) and (g)). Total levels of mTOR (b) and Akt (f) were not significantly altered following stimulation with netrin-1, in either untreated or growth cones treated with cytoskeletal disrupting agents (quantified in (d) and (h)), with the exception of total Akt levels in growth cones treated with colchicine, which showed a small, but significant increase in fluorescence intensity. The number of growth cones analyzed in each treatment group can be found in the corresponding bar of the respective graphs. ${ }^{*} P<0.005$; ${ }^{* *} P<0.0001$ Mann-Whitney test. (i) Pseudocolored images of a live PI3K biosensor (PHAkt-GFP) before and after netrin-1 stimulation in the presence of cytoskeletal inhibitors. Growth cones expressing low levels of PHAkt-GFP were treated with cytoskeletal inhibitors (0.1 $\mathrm{MM}$ cytochalasin D or $12.5 \mu \mathrm{M}$ colchicine) on stage during time-lapse acquisition on an inverted spinning disk confocal system (60x water immersion). Time-lapse imaging showed an increase in PHAkt-GFP signal after netrin-1 stimulation, which was inhibited by cytochalasin D treatment, but not affected by colchicine treatment $(\mathbf{j})$. Background-subtracted fluorescent signals were normalized to the control medium at time 0. Scale bar $10 \mu \mathrm{m}$ 
Author details

${ }^{1}$ Department of Physiology, Development and Neuroscience, University of Cambridge, Downing street, Cambridge CB2 3DY, UK. ${ }^{2}$ Current address: The School of Biomedical Sciences and the Queensland Brain Institute, The

University of Queensland, St Lucia 4072, QLD, Australia. ${ }^{3}$ Current address: Institute of Neuroscience, Chinese Academy of Sciences, Shanghai 200031,

China.

Published online: 19 June 2015

\section{Reference}

1. Piper M, Cheun Lee A, van Horck FPG, McNeilly H, Bo Lu T, Harris WA, et al. Differential requirement of $\mathrm{F}$-actin and microtubule cytoskeleton in cue-induced local protein synthesis in axonal growth cones. Neural Dev. 2015;10:3

\section{Submit your next manuscript to BioMed Central and take full advantage of:}

- Convenient online submission

- Thorough peer review

- No space constraints or color figure charges

- Immediate publication on acceptance

- Inclusion in PubMed, CAS, Scopus and Google Scholar

- Research which is freely available for redistribution 